\title{
Influence of thermal fluctuations on the Nernst signal in superconducting $(\mathrm{K}, \mathrm{Ba}) \mathrm{BiO}_{3}$ single crystals
}

\author{
T. Klein, ${ }^{1,2}$ Z. Pribulova, ${ }^{1,3}$ R. Piquerel, ${ }^{1}$ H. Cercellier,,${ }^{1,2}$ J. Marcus, ${ }^{1}$ and C. Marcenat ${ }^{4}$ \\ ${ }^{1}$ Institut Néel, CNRS, 25 rue des Martyrs, F-38042 Grenoble, France \\ ${ }^{2}$ Université Joseph Fourier, B.P 53, F-3804l Grenoble, France \\ ${ }^{3}$ Centre of Very Low Temperature Physics, Institute of Experimental Physics of the Slovak Academy of Sciences and FS UPJS, \\ Watsonova 47, 04001 Kosice, Slovakia \\ ${ }^{4}$ SPSMS, UMR-E 9001, CEA-INAC/UJF-Grenoble 1, 17 rue des martyrs, F-38054 Grenoble, France
}

(Received 6 December 2010; published 17 March 2011)

\begin{abstract}
We report on the Nernst effect, specific heat and transport measurements performed in high quality $(\mathrm{K}, \mathrm{Ba}) \mathrm{BiO}_{3}$ single crystals close to optimal doping $\left(T_{c} \sim 31 \mathrm{~K}\right)$. We show that a nonzero Nernst effect remains visible well above the upper critical field unambiguously deduced from the onset of the specific heat anomaly. This finite Nernst signal is attributed to fluctuations of the amplitude of the order parameter in a region where the free energy is smaller than $k_{B} T$. Despite the absence of any vortex liquid phase (and hence of any significant phase fluctuations), the field and temperature dependence of the Nernst coefficient is very similar to the one obtained in electron-doped cuprates.
\end{abstract}

DOI: 10.1103/PhysRevB.83.094524

PACS number(s): 74.40.-n, 74.25.Op

\section{INTRODUCTION}

The discovery of an anomalously large Nernst signal above the critical temperature $\left(T_{c}\right)$ in hole-doped cuprates ${ }^{1-5}$ rapidly imposed this technique as a fundamental probe for the study of superconducting fluctuations in type II superconductors. As no qualitative difference could be observed between the low $\left(T<T_{c}\right)$ and high $\left(T>T_{c}\right)$ temperature regimes, this anomalous signal has been attributed to the existence of a vortex-like excitation (i.e., phase fluctuations) above the superconducting transition. This behavior supported the idea that $T_{c}$ could correspond to the loss of long-range phase rigidity of Cooper pairs pre-existing in the so-called pseudogap state (see also Refs. 6-10 for theoretical developments on the Nernst effects in cuprates). Similarly, in the vicinity of a superconductor-insulator transition, the very low carrier density is also expected to give rise to strong phase fluctuations, accounting, for example, for the nonzero Nernst effect that has been observed above $T_{c}$ in $\mathrm{InO}_{x}$ (Ref. 11).

This scenario was further supported by experiments in electron-doped cuprates for which there is no evidence for any pseudogap and for which the Nernst signal vanishes at $T_{c}$. However, besides this fundamental difference, the field dependence of the Nernst effect for $T<T_{c}$ is very similar to the one observed in hole-doped samples. Indeed, in all systems the Nernst effect rapidly increases above the irreversibility line [for which the critical current vanishes], reaching a maximum for intermediate fields, and progressively decreases at high fields. This "tilted hill" profile has hence been attributed to the wandering of the vortex lines associated with the melting of the vortex solid and the upper critical field is defined as the field for which the Nernst effect is getting equal to zero.

To probe this scenario, we have performed specific heat, transport, and Nernst effect measurements in $(\mathrm{K}, \mathrm{Ba}) \mathrm{BiO}_{3}$ single crystals. This system presents some similarities with cuprates since superconductivity ${ }^{12}$ is also obtained by doping of a nonconventional insulating phase resulting here from the charge ordering of bismuth..$^{13}$ However, the description of its superconducting properties is easier. First, tunneling spectroscopy measurements reveal a classical BCS-type superconducting gap ${ }^{14}$ and no evidence for an unconventional coupling mechanism has been reported so far. Second, due to its cubic (i.e., isotropic) structure, this system is a powerful tool to investigate the influence of dimensionality on the superconducting properties (see, for instance, Refs. 15 and 16). Third, thermal fluctuations are more than two orders of magnitude smaller than in cuprates.

This latter point itself has several important consequences. First, no liquid phase can be observed in this system and phase fluctuations are hence irrelevant. ${ }^{17}$ The irrelevance of vortex line wandering has further been inferred from the functional dependence of the torque signal on temperature and field in heavy ion irradiated samples. ${ }^{16}$ Moreover, if strong fluctuations hinder any direct determination of the upper critical field $\left(H_{c 2}\right)$ in cuprates, $(\mathrm{K}, \mathrm{Ba}) \mathrm{BiO}_{3}$ presents the great advantage of showing clear specific heat anomalies from which $H_{c 2}$ can be unambiguously deduced. ${ }^{18}$ It is hence possible to compare the field for which the Nernst signal vanishes to this $H_{c 2}$ line.

However, it is important to note that thermal fluctuations are not fully negligible in $(\mathrm{K}, \mathrm{Ba}) \mathrm{BiO}_{3}$ and we have then shown ${ }^{19}$ that the (pinning dependent ${ }^{17}$ ) position of the $H_{c 2}$ line is obtained by assuming that the difference between the free energy densities in the normal and superconducting states $\left[\Delta F=F_{N}-F_{S}(H)\right]$ becomes on the order of $k_{B} T / V_{\text {coh }}$ for $H=H_{c 2}$ :

$$
\Delta F=\left(\mu_{0} H_{c}^{2} / 2\right)\left(1-H_{c 2} / H_{c 2}^{M F}\right)^{2} \sim k_{B} T / V_{\mathrm{coh}}
$$

where $V_{\text {coh }}$ is the correlation volume and $H_{c 2}^{M F}$ the mean field critical field. The upper critical field is hence shifted towards lower temperature. The amplitude of the order parameter is then small for $H_{c 2}^{M F}>H>H_{c 2}$ and fluctuations in this amplitude are expected to be important in this region. The main result of the present work is to show that a non zero Nernst signal remains visible well above the $H_{c 2}$ line and that this signal only vanishes for $H \rightarrow H_{c 2}^{M F}\left(>H_{c 2}\right)$. 


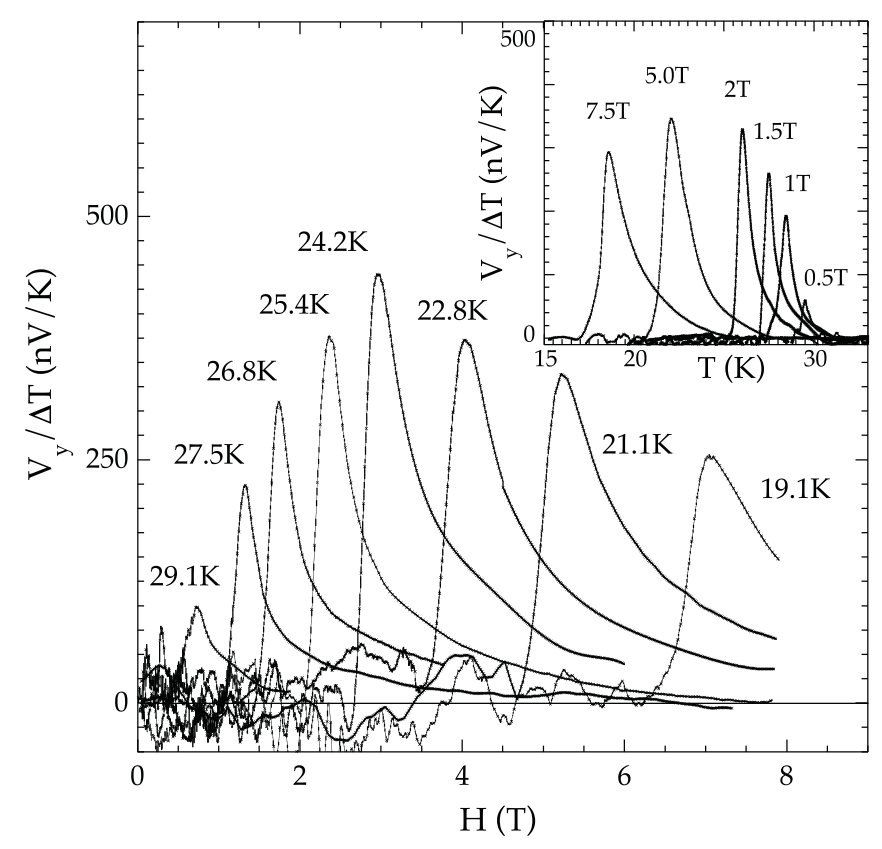

FIG. 1. Nernst signal $\left(e_{N}=V_{y} / \Delta T\right)$ in the presence of a heat gradient $(\Delta T \sim 1 \mathrm{~K})$ along the $x$ direction a function of applied magnetic field (along the $z$ direction) for the indicated temperatures in a $(\mathrm{K}, \mathrm{Ba}) \mathrm{BiO}_{3}$ single crystal. Inset: $V_{y} / \Delta T$ as a function of the temperature for the indicated values of the field.

\section{SAMPLES AND EXPERIMENTS}

The measurements have been performed in a high quality $\left(\mathrm{K}_{x}, \mathrm{Ba}_{1-x}\right) \mathrm{BiO}_{3}$ single crystal close to optimal doping $(x \sim$ $\left.0.35, T_{c} \sim 31 \mathrm{~K}\right)$. Those crystals display very sharp transitions in both transport and magnetic susceptibility $\left(\triangle T_{c}<0.2 \mathrm{~K}\right)$ as well as very well-defined specific heat anomalies [see Fig. 2(b) and Ref. 17]. The Nernst effect is the detection of a transverse voltage $\left(V_{y}\right)$ when a heat gradient is applied along the $x$ direction in the presence of a perpendicular magnetic field $B_{z}: e_{N}=E_{y} / \nabla T_{x} \quad\left(=V_{y} / \triangle T_{x}\right.$ for square samples). Those measurements have been performed on a $\sim 1 \times 1 \times 0.3 \mathrm{~mm}^{3}$ slice cut out of a single crystal. Three thermocouples have been placed on both ends and in the middle of the sample. A heat gradient of about $1 \mathrm{~K}$ was applied to the sample by heating up one edge of the slice. The reported temperature was measured at the center of the sample and we checked that the heat gradient was linear along the sample. The specific heat measurements were previously performed on single crystals from the same batch using an ac technique as described in Ref. 18 and the transport measurements were done using a standard four probe geometry.

\section{RESULTS AND DISCUSSION}

As no Nernst effect is observed above $T_{c}$ for $H=0,{ }^{20}$ the superconducting contribution to $e_{N}$ can be directly identified without any further treatment. As observed in cuprates, $e_{N}$ is very small at low field and low temperature (see Fig. 1) due to pinning hindering the vortex motion in the vortex solid state. In $(\mathrm{K}, \mathrm{Ba}) \mathrm{BiO}_{3}$, the irreversibility line can be deduced from both $R \rightarrow 0$ and from the onset of the diamagnetic response. This line lies close to the midpoint of the specific

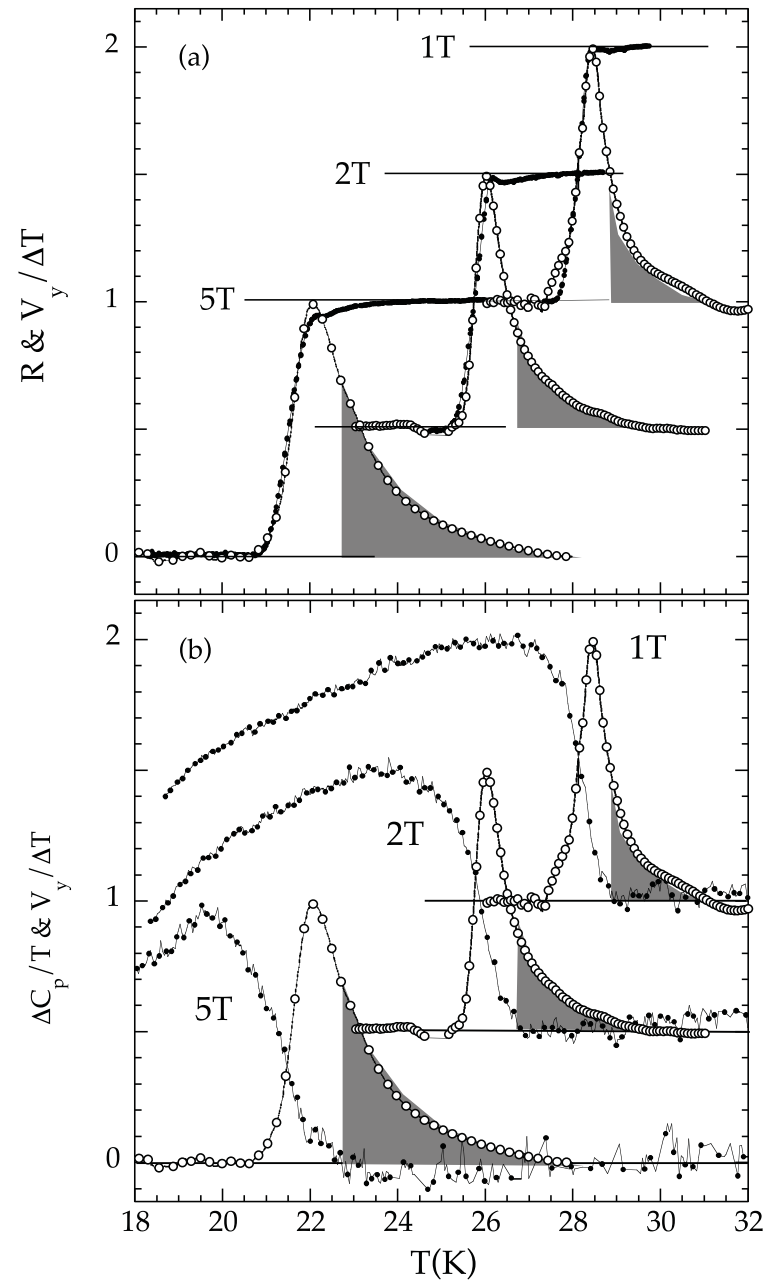

FIG. 2. (a) Renormalized Nernst signal ( $V_{y} / \Delta T$, open circles) and resistivity ( $R$, thick line) as a function of the temperature for the indicated values of the magnetic field in $(\mathrm{K}, \mathrm{Ba}) \mathrm{BiO}_{3}$ single crystals. (b) Nernst signal $\left(V_{y} / \Delta T\right.$, open circles) and electronic contribution to the specific heat anomaly $\left(\Delta C_{p} / T\right.$, closed circles) as a function of the temperature for the indicated values of the magnetic field. The shaded area indicates the region for which a nonzero Nernst effect is observed above the onset of the specific heat anomaly. The curves at 2 and $1 \mathrm{~T}$ have been shifted for clarity.

heat anomaly, ${ }^{18}$ and as shown in Fig. 2(b), the onset of $e_{N}$ lies close to this $C_{p}$ midpoint. $e_{N}$ then rapidly increases above the irreversibility line following the $R$ versus $T$ curve [Fig. 2(a)]. Indeed, $e_{N}$ is related to the electrical $\left(\sigma_{i j}\right)$ and thermoelectrical $\left(\alpha_{i j}\right)$ components of the conductivity tensor through $e_{N}=\left[\alpha_{x y} \sigma_{x x}-\alpha_{x x} \sigma_{x y}\right] /\left[\sigma_{x x}^{2}+\sigma_{x y}^{2}\right]$ and in the absence of significant Hall effect $e_{N}=\rho \alpha_{x y}$. Neglecting the small change in $\alpha_{x y}$ on the narrow temperature (or field) range of the transition, one therefore directly gets $e_{N} \propto \rho$ in the close vicinity of the irreversibility line, in good agreement with the data. In the standard mean-field theory the Nernst effect is then expected to decrease linearly towards zero for $H \rightarrow H_{c 2}:{ }^{10,21} e_{N}^{M F} \sim \rho \times\left[H-H_{c 2}^{M F}(T)\right] /\left(4 \kappa^{2} T\right)$. On the contrary, in $(\mathrm{K}, \mathrm{Ba}) \mathrm{BiO}_{3} e_{N}$ does not vanish for $H=H_{c 2}$ but remains clearly finite for fields well above the onset of the specific heat anomaly [shaded areas in Fig. 2(b)]. 


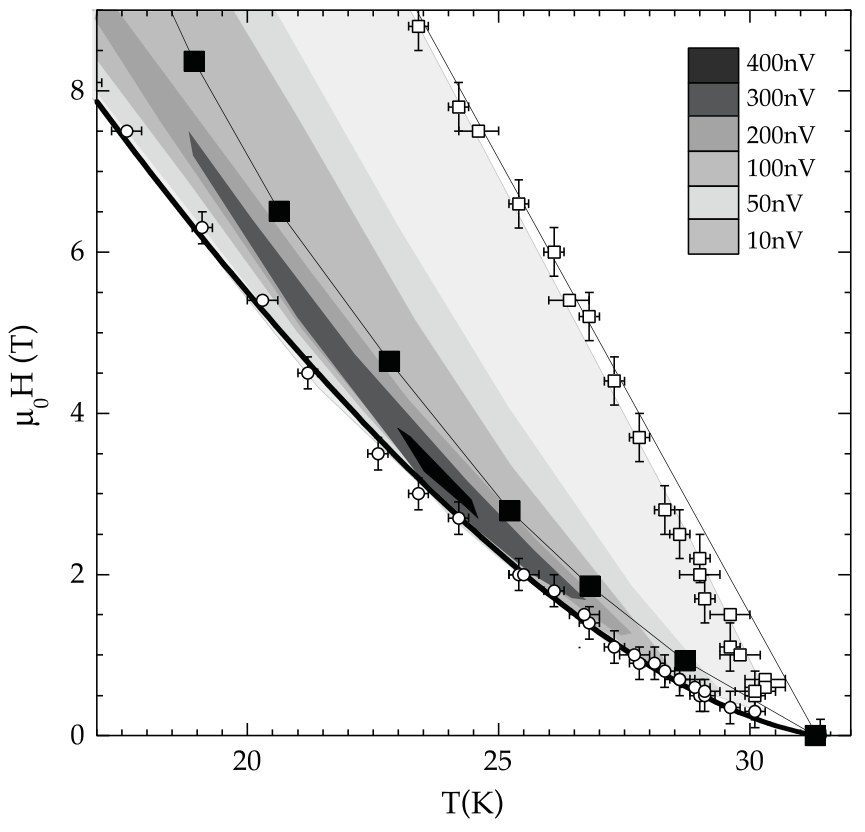

FIG. 3. $H-T$ phase diagram of $(\mathrm{K}, \mathrm{Ba}) \mathrm{BiO}_{3}$ single crystals. The closed squares correspond to the upper critical field deduced from the onset of the specific heat anomaly (Fig. 2). The open circles (or squares) correspond to the onset (or end point) of the Nernst signal. The thick solid line is a $(1-t)^{1.5}$ fit corresponding to the irreversibility line. The gray scale corresponds to regions with successively higher values of $e_{N}$ (contour plots).

By analogy to this standard mean field theory, we have reported in the inset of Fig. 4 (open symbols) both $e_{N}(T)$ and the value of $\rho \times\left[H-H_{c 2}(T)\right] /\left(4 \kappa^{2} T\right)$ [taking $\kappa \sim 100$ (Ref. 22)] where $H_{c 2}$ is now the upper critical field deduced from the $C_{p}$ anomaly i.e. rescaled by the fluctuations. As shown, this simple formula reproduces very well quantitatively the experimental data for $T<T_{c 2}(H)$ (a similar agreement is obtained for the other magnetic fields) but, in contrast to the simple mean field scenario, for $H \neq 0, e_{N}$ remains finite for $T_{c 2}<T<T_{c 2}^{M F}$ [or equivalently for $H_{c 2}(T)<H<H_{c 2}^{M F}$ ] due to fluctuations in the amplitude of the order parameter in the region where $0<\Delta F<k_{B} T / V_{\text {coh }}$. Note that, as discussed in (Ref. 19), the thermal fluctuations are also expected to lead to a small rescaling of the zero field $T_{c}$ (typically on the order of $1 \mathrm{~K})$. However as the maximum of the signal scales as $\left.H^{2} / T\right)^{1 / 3}$ (see below) we were unable to probe this rescaling in our data for $H \rightarrow 0$.

The continuity of the Nernst signal through the $H_{c 2}$ line is further emphasized by the contour plots displayed in Fig. 3. We have reported on this $\mathrm{H}-\mathrm{T}$ diagram, the lines corresponding to the onset (open circles) and end ( $e_{N}<1 \mathrm{nV} / \mathrm{K}$, open squares) of the Nernst effect, together with this thermodynamic $H_{c 2}$ line (closed squares). The gray scale corresponds to regions with successively higher values of $e_{N}$ (contour plots). As shown, no peculiar feature is observed for $e_{N}$ as $H$ crosses the $H_{c 2}$ line. Note that the slope of the line corresponding to the end of the Nernst signal is on the order of $1.2 \mathrm{~T} / \mathrm{K}$ being close to the value observed in heavily irradiated samples ${ }^{17}$ leading to $\mu_{0} H_{c 2}(0) \sim 0.7 \times 1.2 \times T_{c} \sim 26 \mathrm{~T}$ in good agreement

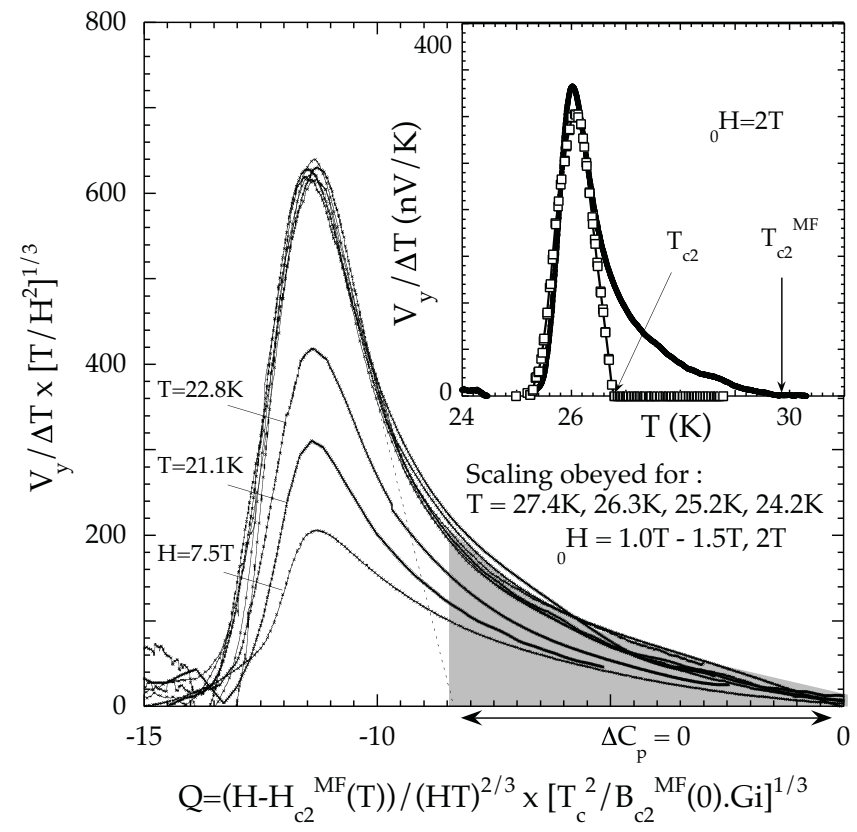

FIG. 4. LLL scaling of the Nernst coefficient in $(\mathrm{K}, \mathrm{Ba}) \mathrm{BiO}_{3}$ single crystals. (The shaded area corresponds to the region above the onset of the specific heat anomaly). Inset: temperature dependence of the Nernst effect at $2 \mathrm{~K}$ (full line) in comparison to $\rho[B-$ $\left.B_{c 2}(T)\right] /\left(4 \mu_{0} \kappa^{2} T\right)$ (open squares, see text for details).

with the value deduced from magnetotransport data at low temperature $^{23}\left(H_{\mathrm{irr}} \rightarrow H_{c 2}\right.$ for $T \rightarrow 0$ ).

In heavy ion irradiated samples, the torque signal presents a discontinuous jump $\left(\Gamma_{0}\right)$ when the magnetic field is aligned with the amorphous tracks. In $(\mathrm{K}, \mathrm{Ba}) \mathrm{BiO}_{3}, \Gamma_{0} / T B$ then scales as $|Q|^{3 / 2}$ (Ref. 16) where $Q$ is the Lowest Landau Level (LLL) parameter: $\left[\left(H_{c 2}^{M F}(T)-H\right) /(H T)^{2 / 3}\right] \times$ $\left[\left(T_{c}^{2} / H_{c 2}^{M F}(0) G_{i}\right)^{1 / 3}\right]$ with $G i \equiv \frac{1}{2}\left[k_{B} T_{c} / \varepsilon_{0} \xi\right]^{2} \sim 3 \times 10^{-4}$ $\left(\varepsilon_{0}=\left(\Phi_{0} / 4 \pi \lambda\right)^{2}\right.$ being the vortex line energy, $\lambda$ the penetration depth and $\xi$ the coherence length ${ }^{24}$ ). To check the validity of this LLL scaling on the Nernst signal, we have plotted in Fig. $4 e_{N} \times\left(T / H^{2}\right)^{1 / 310}$ as a function of $Q$ where $Q$ has been calculated assuming that $H_{c 2}^{M F}$ lies close the end point of the Nernst effect (thin solid line in Fig. 3).

As shown in Fig. 4, this scaling is very well obeyed for fields smaller than $\sim 3 \mathrm{~T}$ and/or temperature larger than $\sim 24 \mathrm{~K}$. All the characteristic lines are then defined by specific values of $Q(T, H)$. As pointed out by van der Beek et al. ${ }^{16}$ Eq. (1), which defines $H_{c 2}$, is equivalent to $Q=-[4 \pi \sqrt{2}]^{2 / 3} \sim-7$ if one assumes that $V \sim a_{0}^{2} \xi, a_{0}$ being the intervortex spacing. We here obtain that the onset of the $C_{p}$ lies close to $Q=-8$, in good agreement with this estimation. Similarly, one can asser ${ }^{25}$ that the irreversibility line in disordered systems is also given by some peculiar value of $Q(T, H)$. As shown in Fig. 4 , the onset of the Nernst signal at the irreversibility line here corresponds to $C \sim-13$ (as a comparison, $|C|$ values on the order of 11-15 have been recently inferred for the irreversibility line in iron pnictides ${ }^{25}$ ). The scaling fails at high field/low temperature, but this decrease in the amplitude is probably related to the broadening of the resistive transition (i.e., $\rho<\rho_{N}{ }^{26}$ ). 


\section{SUMMARY AND CONCLUSIONS}

We therefore obtain the following scenario: The $H_{c 2}$ line in the $(\mathrm{K}, \mathrm{Ba}) \mathrm{BiO}_{3}$ system is shifted toward lower temperatures due to [small but not negligible] thermal fluctuations. In a LLL scaling scenario this line is then defined by $Q \sim-8$ corresponding to the line for which the condensation energy becomes on the order of $k_{B} T / a_{0}^{2} \xi$. A peak of the Nernst effect is observed in the vicinity of this rescaled $H_{c 2}$ line, but $e_{N}$ remains finite up to $H_{c 2}^{M F}$. Finally we would like to emphasize the similarity between the temperature and field dependence of the Nernst signal observed here and the one previously obtained in electron doped cuprates. Indeed, despite the irrelevance of vortex wandering in our system, the field (and/or temperature) dependence of $e_{N}$ has the characteristic "tilted-hill" profile previously observed in cuprates (see Fig. 1 (present work) and Fig. 27 in Ref. 3). Moreover the similarity in the contour plots of those two systems is striking (see Fig. 3 (present work) and Fig. 28 in Ref. 3) and it would hence be interesting to test this scenario in electron doped cuprates.
${ }^{1}$ Z. A. Xu, N. P. Ong, Y. Wang, T. Kakeshita, and S. Uchida, Nature (London) 406, 486 (2000).

${ }^{2}$ Y. Wang, N. P. Ong, Z. A. Xu, T. Kakeshita, S. Uchida, D. A. Bonn, R. Liang, and W. N. Hardy, Phys. Rev. Lett. 88, 257003 (2002); Y. Wang, S. Ono, Y. Onose, G. Gu, Y. Ando, Y. Tokura, S. Uchida, and N. P. Ong, Science 299, 86 (2003).

${ }^{3}$ Y. Wang, L. Li, and N. P. Ong, Phys. Rev. B 73, 024510 (2006), and references therein.

${ }^{4}$ C. Capan, K. Behnia, J. Hinderer, A. G. M. Jansen, W. Lang, C. Marcenat, and C. Marin, and J. Flouquet, Phys. Rev. Lett. 88, 056601 (2002).

${ }^{5}$ J. Chang, R. Daou, C. Proust, D. LeBoeuf, N. Doiron-Leyraud, F. Lalibert, B. Pingault, B. J. Ramshaw, R. Liang, D. A. Bonn, W. N. Hardy, H. Takagi, A. B. Antunes, I. Sheikin, K. Behnia, and L. Taillefer, Phys. Rev. Lett. 104, 057005 (2010).

${ }^{6}$ R. Daou, J. Chang, D. Leboeuf, O. Cyr-Choinière, N. DoironLeyraud, B. J. Ramshaw, R. Liang, D. A. Bonn, W. N. Hardy, and L. Taillefer, Nature (London) 463, 519 (2010).

${ }^{7}$ D. Podolsky, S. Raghu, and A. Vishwanath, Phys. Rev. Lett. 99, 117004 (2007).

${ }^{8}$ B. D. Tinh and B. Rosenstein, Phys. Rev. B 79, 024518 (2009).

${ }^{9}$ S. Mukerjee and D. A. Huse, Phys. Rev. B 70, 014506 (2004).

${ }^{10}$ S. Ullah and A. T. Dorsey, Phys. Rev. B 44, 262 (1991); Phys. Rev. Lett. 65, 2066 (1990).

${ }^{11}$ P. Spathis, H. Aubin, A. Pourret, C. Capan, and K. Behnia, Europhysics. Lett. 83, 57005 (2008).

${ }^{12}$ M. D. Nunez Regueiro and A. A. Aligia, Phys. Rev. Lett. 61, 1889 (1988).

${ }^{13}$ C. H. Du and P. D. Hutton, Europhys. Lett. 31, 145 (1995); R. M. Noack, D. J. Scalapino, and R. T. Scalettar, Phys. Rev. Lett. 66, 778 (1991).
${ }^{14}$ P. Szabo, P. Samuely, A. G. M. Jansen, J. Marcus, and P. Wyder, Phys. Rev. B 62, 3502 (2000).

${ }^{15}$ T. Klein, I. Joumard, S. Blanchard, J. Marcus, R. Cubitt, T. Giamarchi, and P. Ledoussal, Nature (London) 413, 404 (2001).

${ }^{16}$ C. J. van der Beek, M. Konczykowski, L. Fruchter, R. Brusetti, T. Klein, J. Marcus, and C. Marcenat, Phys. Rev. B 72, 214504 (2005).

${ }^{17}$ C. Marcenat, S. Blanchard, J. Marcus, L. M. Paulius, C. J. van der Beek, M. Konczykowski, and T. Klein, Phys. Rev. Lett. 90, 037004 (2003).

${ }^{18}$ S. Blanchard, T. Klein, J. Marcus, I. Joumard, A. Sulpice, P. Szabo, P. Samuely, A. G. M. Jansen, and C. Marcenat, Phys. Rev. Lett. 88, 177201 (2002)

${ }^{19}$ T. Klein, C. Marcenat, S. Blanchard, J. Marcus, C. Bourbonnais, R. Brusetti, C. J. van der Beek, and M. Konczykowski, Phys. Rev. Lett. 92, 037005 (2004).

${ }^{20}$ Despite the proximity of a metal insulator transition, the $e_{N} / T B$ value can be estimated to be lower than $210^{-2} \mathrm{nV} / \mathrm{TK}^{2}$ [following K. Behnia, M. A. Measson, and Y. Kopelevich, Phys. Rev. Lett. 98, 076603 (2007)].

${ }^{21}$ K. Maki, Physica 55, 124 (1981).

${ }^{22}$ I. Joumard, J. Marcus, T. Klein, and R. Cubitt, Phys. Rev. Lett. 82, 4930 (1999).

${ }^{23}$ P. Samuely, P. Szabo, A. G. M. Jansen, T. Klein, C. EscribeFilippini, and P. Wyder, Europhys. Lett. 41, 207 (1998).

${ }^{24}$ Note that this number quantifies the strength of thermal fluctuations and is on the order of $\sim 4 \times 10^{-2}$ in $\mathrm{YBaCuO}$.

${ }^{25}$ J. Kacmarcik, C. Marcenat, T. Klein, Z. Pribulova, C. J. van der Beek, M. Konczykowski, S. L. Budko, M. Tillman, N. Ni, and P. C. Canfield, Phys. Rev. B 80, 014515 (2009).

${ }^{26}$ Following, ${ }^{10} \alpha_{x y} \times\left[T / H^{2}\right]^{1 / 3}$ is expected to scale with $Q$ and we have here assumed that $e_{N}=\rho_{N} \alpha_{x y}$ as $\rho$ is close to its normal state value above the peak of the Nernst coefficient. 